\title{
Accelerating Antibody Discovery Using Transgenic Animals Overexpressing the Neonatal Fc Receptors As A Result Of Augmented Humoral Immunity
}

\author{
Imre Kacskovics \\ Department of Immunology, Eötvös Loránd University, \\ Budapest and ImmunoGenes Ltd
}

We have developed transgenic ( $\mathrm{Tg}$ ) mice and rabbits that overexpress the neonatal Fc receptor (FcRn) in tissue and cell specific manner. Notably, cells that are important in protecting IgG from degradation and in antigen presentation express FcRn at a significantly higher level in these animals than in wild type ones. As a result, at the beginning of a secondary humoral immune response when switching from IgM to IgG is occurring, the overexpressed FcRn provides better IgG protection in capillary endothelial cells and hemopoietic cells leading to higher level of circulating antigenspecific IgG. This leads to an elevated amount of antigen-IgG immune complexes (IC) during the secondary immune response which enhances IC transport into lymphoid organs and antigen presentation via the Fc $\gamma$ Rs. In addition, and independently, FcRn overexpressed in antigen presenting cells facilitates more efficient transport of antigen-IgG IC into lysosomes. The improved antigen presentation leads to the activation of more antigen-specific helper $\mathrm{T}$ cells and eventually many more antigen-specific B-cells in lymphoid organs. Thus the combined effect of these different factors results in an augmented humoral immune response. Consequently, FcRn overexpression leads to a number of practical benefits for improved generation of monoclonal and polyclonal antibodies against multiple antigens, including weakly immunogenic epitopes, like G-protein related receptors (GPCRs) or ion channels. 